\section{«Les Païens de la Pléiade»: L'Érotisme dans les Folastries de Ronsard et dans les Gayetez d'Olivier de Magny}

DAVID DORAIS

Summary: This article compares the eroticism of Ronsard's Folastries (1553) with that of the Gayetez (1554) of Olivier de Magny. Although the former exercised some influence on the latter, the eroticism of the two poets turns out to be very different, as much in thematic and stylistic terms as with regard to vocabulary. Moreover, on the conceptual level, when it comes to depicting the body and erotic technique, Magny proves to be far more chaste and less risqué than his master. Nevertheless, the two share at once a belief in the aphrodisiac power of poetry and a mistrust of the sexual act itself.

«Les païens de la Pléiade »... L'expression vient de Paul Laumonier qui, dans l'introduction au cinquième tome des Euvres complètes de Ronsard, parle de l'influence qu'eut le livret des Folastries sur « tous les 'païens' de la nouvelle école, entre autres Baïf et Muret, auxquels il était dédié, Tahureau, Magny et la Péruse, qui l'imitèrent immédiatement »1. C'est donc que les Folastries se placeraient à l'origine de toute une lignée d'œuvres lestes — « mignardises », « gayetez — - qui, qualifiées de « païennes », se plairaient à évoquer les voluptés de la chair, faisant fi d'une mauvaise conscience héritée du christianisme. Cet érotisme ronsardien n'a, selon nous, été étudié que rarement et, à plus forte raison, il a été négligé chez ces autres auteurs censés avoir été séduits par les Folastries. Nous nous proposons donc ici d'étudier l'érotisme dans le Livret de 1553 et de le comparer avec celui des Gayetez d'Olivier de Magny, recueil publié un an plus tard ${ }^{2}$.

Cependant, cet érotisme qui représente l'objet principal de notre curiosité ${ }^{3}$ est-il bien l'enjeu majeur des deux œuvres analysées? À l'examen, il 
apparaît que la réponse est négative. C'est, en effet, une caractéristique importante de l'érotisme au XVI ${ }^{\mathrm{e}}$ siècle que de ne s'être pas encore constitué en genre littéraire à part entière ; on ne peut pas encore parler de récit ou de poésie érotique autonomes. L'érotisme est un thème libre et bienvenu dans toutes les œuvres de style bas, bucoliques, satires ou épigrammes, par exemple, mais on ne retrouve pas encore à la Renaissance française cette tension qui sera celle du roman libertin du XVIII ${ }^{\mathrm{e}}$ siècle, consacré tout entier à la mise en scène des ébats amoureux. Pour certains historiens ${ }^{4}$, la littérature pornographique moderne, littérature marginale et de transgression, ne sera créée qu'en réaction à l'établissement d'une forte censure morale, c'est-àdire à partir de la Réforme et de la Contre-Réforme, puis surtout avec le classicisme. De même, pour Jean-Marie Goulemot, spécialiste de la littérature érotique, " chez Rabelais et les hommes de la Renaissance ou du préclassicisme, [la littérature érotique] voisinait avec les formes admises de la pratique littéraire, en une espèce de naturel et d'innocence gaillarde, qui faisait de sa lecture un acte culturel reconnu et accepté „5. L'érotisme à la Renaissance se module selon l'esthétique du mélange, ne structurant pas à lui seul des recueils entiers, mais inspirant plutôt certaines pièces légères qui se trouvent à côtoyer d'autres pièces d'inspiration toute différente. Ainsi, dans les Folastries, les odelettes franchement grivoises voisinent avec des pièces bachiques (VII, VIII, les Dithyrambes), mignardes (V, VI), parodiques (II) ou comiques et satiriques (les Épigrammes). Le cas est encore plus flagrant dans les Gayetez, où Magny parle de sensualité, mais loue également des personnages politiques contemporains, chante sa haute conception de la poésie ou célèbre le chien de sa dame.

\section{La fonction de l'érotisme}

Mais dans les pièces plus gauloises des deux auteurs, quelle est la fonction de l'érotisme? Quelle signification, quelle orientation particulière donne-t-il aux textes? Cette fonction change selon chacun des auteurs. En effet, chez Ronsard, l'érotisme joue un rôle beaucoup plus important que chez Magny. Tout d'abord, il sert à exprimer chez le Vendômois une nouvelle conception de l'amour, toute différente de celle des Amours de 1552 et qui préfigure, tout en s'en distinguant, la conception qui sera celle de la Continuation de 1555. Tout différent de l'adoration pétrarquiste qui se voulait idéalisée et contemplative, l'amour exprimé dans les Folastries s'apparente plutôt à un désir charnel criant. Curieusement, Ronsard, " poète de la conquête amoureuse ", se heurte ici à une série de situations d'échec où l'union charnelle 
se voit toujours frustrée. La « pucelette » qu'il convoite se trouve successivement, dans les odes I, II, III, V et VI, dérobée à son étreinte par un « Tyran de village », par « deux Soldars aventuriers », par Catin la bigote, par une famille alertée par un chien et par un « enfant quartannier ». C'est pourquoi les deux temps de conjugaison les plus courants dans les Folastries sont l'indicatif présent et le passé : soit la possession physique a déjà eu lieu et est maintenant perdue, soit le poète est, au moment où il parle, en train de se faire contester son ascendant sur les jeunes filles. Quelquefois le futur (« Sans vous je garderay bien / Vos sœurs » [II, v. 82-83]) ou le subjonctif couplé au conditionnel («Que pleust à dieu que je peusse / Pour un soir devenir puce, / [...] / Sans soupson je coucheroy / Entre tes bras, ma cruelle » [VI, v. 35-36, 42-43]) viennent introduire une note d'espoir. Mais en général, l'amour, loin d'être une admiration lointaine, prend la forme d'un désir de proximité toujours déjoué6. Il s'agit d'une pulsion sensuelle croissante et jamais satisfaite, d'autant plus frustrante que l'évocation du corps féminin, lui, se fait sans ambages ${ }^{7}$.

Cette nouvelle conception de l'amour reposant sur l'érotisme joue un autre rôle dans les Folastries. Selon nous, cette expression franche de la concupiscence sert à consolider le motif de la sincérité chez Ronsard. Le Livret, qui a heurté les mœurs publiques dès sa publication ${ }^{8}$, veut; par l'affirmation de désirs plus terre-à-terre, par l'imposition d'un ton licencieux, donner l'impression d'une prise de parole plus personnelle. Après a voir adopté le détachement pétrarquiste, Ronsard, par sa conversion au style bas qui s'amorce dans les Folastries, veut endosser une nouvelle persona, il veut donner au locuteur une apparence plus convaincante de sincérité, d'authenticité, de naturel.

Dans les Gayetez de 1554, l'érotisme de Magny joue un rôle moins structurant. Il sert plutôt à illustrer d'autres grands thèmes déjà traités dans les Amours publiés un an plus tôt : la poésie, l'amitié et, au premier chef, l'amour. De quelle manière l'érotisme se joint-il ici à l'amour ? Les situations amoureuses peintes par Magny relèvent encore en grande partie du schéma pétrarquiste : l'amant se plaint sur le mode élégiaque de la cruauté de sa dame, de cette " pucelette divine, / Qui [lui] ard le cœur et la poitrine " (XVI, v. 7-8) ; ainsi, dans l'odelette III, l'échec du baiser sert à montrer la froideur de la dame, qui repousse l'amant trop fougueux. L'objet de désir, comme chez Ronsard, reste insaisissable, et c'est pourquoi le mode de conjugaison le plus courant dans la poésie érotique de Magny, par exemple dans l'ode VI A s'amie, est l'impératif, mode qui indique une tension entre 
l'homme et la femme, le désir de domination d'un objet qui ne nous appartient pas encore. Dans d'autres cas, le temps futur sert à donner forme à l'espoir que l'amour et l'érotisme survivront dans les Champs-Élysées : "Là nous irons, là noz douces amours / Doucettement ensemble conduyrons, /Et d'un plaisir ensemble joüyrons, / D'un doux plaisir qui durera tousjours » $(G ., \mathrm{XXX}, \mathrm{v} .57-60)$. Mais très rarement l'amant parle-t-il au passé, comme dans l'ode $\mathrm{XVI}^{9}$ : au mieux, il peut passer à l'indicatif et au participe présent, tous deux concentrés dans l'immédiat de la sensualité, le premier évoquant plutôt l'instantanéité du plaisir, le second, le déroulement d'un geste dans un court laps de temps.

Cependant, Magny, moins aventureux que Ronsard, affirme rarement avec véhémence ses élans charnels, il hésite à quitter le giron pétrarquiste pour aller imiter comme son maître la poésie latine hendécasyllabique inspirée de Catulle ; il utilise simplement l'érotisme pour illustrer l'amour conventionnel que Ronsard cherchait à rejeter.

C'est peut-être à cause de ce schéma élégiaque omniprésent que Alistair MacKay, l'éditeur des Gayetez, a pu dire qu'elles n'étaient « ni érotiques ni réalistes » et qu'elles adoptaient "un certain ton sérieux " ${ }^{10}$. De même, François Rouget dit que « seul l'éloignement caractérise cette relation bien fragile » entre l'amant et sa dame, et que « le style mignard simule habilement ou tente de combler le manque d'une familiarité conquise avec l'interlocutrice ${ }^{11}$. Tandis que, chez Ronsard, l'érotisme constituait une nouvelle orientation thématique, stylistique et éthique, il n'est chez Magny guère plus qu'un motif chargé de donner corps au thème de l'amour pétrarquiste. Il faut, par contre, souligner que, malgré une thématique à demi mélancolique, le ton reste léger, grâce à l'ode courte de sept ou huit syllabes imitée de Ronsard ${ }^{12}$.

\section{Le langage érotique}

La fonction de l'érotisme étant différente chez les deux auteurs, il en découle que cette différence déterminera la représentation de l'acte sexuel dans chacun des deux recueils. Il convient à ce propos de faire quelques remarques sur le langage. Chez Ronsard, le vocabulaire érotique se caractérise surtout par son prosaïsme : plusieurs parties du corps sont énumérées tout au long des poèmes, toujours avec le mot exact. Dans un souci de clarté dicté par le motif de la sincérité, le poète veut nous faire comprendre immédiatement quel est le référent des mots qu'il utilise, tels le «flanc» (I, v. 77, 103), la « cuisse » (I, v. 79), la « fesse » (I, v. 87), la « bouche » (I, v. 133) ou les 
« lèvres » (I, v. 134). Cependant, dans le cas du sein, l'étroitesse du vocabulaire direct est corrigée par la variété des dénominations et l'on peut parler tour à tour de l' « estomac » (I, v. 47, 165 ; III, v. 89), de la « poitrine » (I, v. 88), des «tetins » (I, v. 102 ; III, v. 130), des « mammelles » (III, v. 14, 49 ; VI, v. 55) ou de la « tette » (VI, v. 24). Toutefois, quand il s'agit de parler des genitalia, la stratégie change légèrement : au lieu d'utiliser le terme exact, Ronsard préfère utiliser des sens étendus entrés dans la langue, donc parfaitement compréhensibles pour le lecteur de l'époque. En effet, le sens libre de mots tels que « motte » (colline, tertre), « fossette » (petite fosse) ou « Tribart » (bâton) est attesté dans les dictionnaires de langue ancienne. Ainsi, ce que cherche Ronsard, par l'utilisation du registre dénotatif, c'est moins de choquer ou de stimuler par l'utilisation d'un vocabulaire grossier que de créer une impression d'authenticité et de proximité entre le lecteur et le texte par l'évocation du corps au naturel ${ }^{13}$.

Magny également professe sa foi en la nature, contre l'artifice pétrarquiste : « Je hai de baiser ces marbres, / Ces peintures et ces arbres / Transformez en mile lieux / En mile images des dieux. / Ta seule bouche m'apaste, / Ta seule bouche me flate » (VI, v. 41-46). Mais malgré ce parti pris pour le corps lui-même au détriment de la parure, Magny n'utilise pas de mots crus, il évite les détails précis et réalistes et, tout compte fait, il utilise des métaphores luxueuses, certaines consacrées par Pétrarque, comparant les lèvres à des coraux (III, v. 52), le teint au lis (II, v. 17), ou le sexe féminin à une fleur dédiée à Vénus (XXXI, v. 14) ${ }^{14}$. Malgré ses prétentions au naturel, Magny semble incapable de se distancier du modèle esthétique féminin en vogue dans la poésie de l'époque. Il faut toutefois souligner qu'à une occasion, Magny utilise de belles comparaisons rustiques pour évoquer la dame, la peignant « finement blanche comme laict, / Doucette comme un aignelet, / Et fleurant comme marjoleine » (XXXVIII, v. 31-33) ${ }^{15}$. Il faut également noter que, malgré leurs différences, Ronsard et Magny utilisent tous deux des diminutifs mignards, qui donnent à leurs odelettes un caractère de légèreté et de familiarité.

\section{La représentation du corps}

Inévitablement, la différence de vocabulaire utilisé par les deux poètes entraîne une différence dans la représentation du corps ; alors que chez Ronsard, le corps se veut le plus réaliste possible - l'auteur utilisant très rarement la métaphore - chez Magny, on a moins affaire à un corps de femme qu'à une statue de déesse faite d'ébène, d'ivoire et d'or. Mais au-delà 
de la représentation anatomique proprement dite, comment ce corps est-il mis en scène, quelles postures lui attribue-t-on pour provoquer un effet aguichant? Jean-Marie Goulemot considère que, dans le roman libertin, le procédé du tableau est l'élément-clé du récit érotique. Selon lui, « tout se fonde sur le regard. Il faut donner à voir par l'écriture. Le livre ne peut faire naître le désir de jouissance qu'en décrivant les corps offerts au désir et le stimulant, ou en mettant en scène le tableau des gestes et des attitudes de la jouissance elle-même " ${ }^{16}$. Bien entendu, dans les poésies du XVIe siècle, on ne retrouve pas ces vastes amalgames sadiens qui seront en vogue deux siècles plus tard. La femme est toujours présentée seule et le plus souvent offerte au toucher et au regard de l'amant, qui décrit son maintien et ses gestes.

Ainsi, dans la première Folastrie, le poète compare deux " pucelettes », la « grasselette » et la « maigrelette ». Voulant expliquer pourquoi il aime l'une autant que l'autre, il se met à peindre sous nos yeux le corps passif des deux jeunes filles : « un grasselet enbonpoint, / Une fesse rebondie, / Une poitrine arondie / En deux monteletz bossus... » (I, v. 86-89) pour l'une, tandis que pour l'autre, « elle a les yeux verdeletz / Et les tetins maigreletz. / Son flanc, sa cuisse, sa hanche, / N'ont pas la nege si blanche / Comme a l'autre... » (I, v. 101-5) ${ }^{17}$. Dans d'autres cas, le corps de la pucelle peut se mettre à s'agiter, comme dans la troisième Folastrie, où la " maistresse » du poète, à moitié « bigotée » par Catin, la courtisane repentie qui elle-même, dans sa jeunesse, ne dédaignait pas « le mestier de l'un sus l'autre », veut repousser son amant trop entreprenant. Toutefois, alors qu' on la croirait devenue active, elle se révèle pourtant encore une fois passive, puisque c'est l'auteur qui dicte les mots de son discours ; celui-ci est piégé et, voulant se défendre, la jeune fille ne fait rien d'autre que décrire pour le lecteur les poses suggestives de son corps :

Je ne veux plus que l'on m'acolle.

Pource, ostez vostre main d'abas.

Catin m'a dit qu'il ne faut pas

Que charnelement on me touche,

Halà ma Cousine, il me couche,

Ha ha, lessez, lessez, lessez,

Bran, pourneant vous me préssez, (...)

Ma cuisse en biez accoustrée

Vous defendra tousjours l'entrée,

Et plus les bras vous m'entorsez,

Et plus en vain vous efforcez. (III, 136-42 et 147-50) 
Chez Magny également, la jeune amante est le plus souvent passive. Ainsi, dans l'ode III, le poète la trouve seule au bord d'un ruisseau, abandonnée au sommeil et à moitié nue. Dans l'ode XXV Les Martinales, c'est l'amante de Charbonier, l'un des participants à la bacchanale, qui se trouve soudain frappée par un trait de Cupidon et possédée par la fureur érotique, donnant lieu à l'une des scènes les plus explicites chez Magny, cependant inspirée en grande partie de Jean Second ${ }^{18}$ :

Si bien qu'elle, ainsi attainte,

Soit contraincte

De te requerir pardon,

Te livrant de sa bouchette

Vermeillette

Mille baiseretz en don.

Et t'allechant d'une haleine

Toute pleine

Des parfums de plus grand pris,

De nectar, de miel d'Himette,

De civete,

De canelle et d'ambre gris.

Et puis à ton col branchee,

My-panchee

D'estomac et de menton,

Te laisse en ta bouche tordre,

Voire mordre,

Son petit poil foleton.

Ou chercher de ces pommettes

Les frezettes

Sur l'albastre de son sein,

Ou chercher encor' le reste,

Moins modeste,

D'une fretillante main. (XXV, 121-44)

Enfin, soulignons que chez Ronsard comme chez Magny, l'imagination constitue un autre procédé pour mettre en scène le corps de la dame. Par exemple, dans la Folastrie VI, le poète rêve de se transformer en puce pour surprendre son amie « toute seulette endormie (...) quand plus le doux sommeil / Luy enfleroit la mammelle» (VI, v. 52, 54-55). De même, Magny, lors d'une promenade aux champs, se prend à rêver de sa dame et à souhaiter qu'elle fût là ; «puis », dit-il, « la prenant soubz l'aisselle, / M'en irois 
avecques elle / Dans la forest bien avant » où il lui ferait « une plus douce feste » (XIII, v. 25-27, 29).

\section{L'art érotique}

Au-delà de la mise en scène du corps, les Folastries et les Gayetez offrent un point de vue privilégié sur l'art érotique au XVIe siècle. Pour Michel Simonin, cet ars erotica de la Renaissance se réduit à des « lieux communs toujours récrits » derrière lesquels on retrouve « une pulsion quasi exclusive vers la génitalité, où les autres pratiques ne sont, quand elles sont, tout au plus que des préparations $» 19$. Cette affirmation est surtout vraie pour Ronsard. En effet, celui-ci, bien qu'il laisse place à quelques raffinements comme le simple plaisir de toucher ou d'être serré dans les bras de sa dame, s'occupe surtout à décrire l'accouplement en tant que tel. Encore faut-il amener une précision : si Ronsard déploie son vocabulaire pour peindre le coït, son regard ne se fixe pas que sur la "génitalité ». Pour lui, l'important dans l'acte sexuel n'est pas les membres qui sont mis en jeu, mais bien l'élan, le dynamisme que cet acte entraîne. Parlant de l'union des amants, Ronsard utilisera pour suggérer le mouvement aussi bien des substantifs, comme " manimens fretillars», «tourdions », qui désignent des contorsions, des mouvements de danse, ou " tremoussante souplesse », que des verbes comme « ressecouer », « repousser », « remouvoir », « trepigner », « remuer » ou « tremousser »!

Qui plus est, ce dynamisme inhérent à l'acte sexuel, Ronsard ne le voit pas qu'au niveau microcosmique : il est pour lui le symbole de l'harmonie de l'univers tout entier. C'est ce que suggère la Folastrie IV, pièce entièrement composée sur le modèle de l'équilibre et de la réciprocité. En effet, dans la dernière scène de cette odelette, Jaquet " jauche » Robine et le mouvement ainsi initié se transmet à toute la nature :

Et le bon Jaquet qui l'embroche

Fist trepigner tous les Sylvains

Du dru maniment de ses reins.

Les boucs barbus qui l'agueterent,

Paillars, sur les chevres monterent,

Et ce Jaquet contr' aguignant

Alloient à l'envy trepignant. (IV, 92-98)

Le geste des deux humains est repris et imité par tous les «Sylvains » symbolisant les forces primitives de la nature. L'éros, pour Ronsard, dans une conception proche de celle d'Éryximaque dans le Banquet, est un 
principe universel, une force contagieuse qui se communique au cosmos et en assure l'équilibre.

En comparaison, l'art érotique de Magny est beaucoup plus chaste. Rarement évoque-t-il le " dernier point », se contentant de plaisirs plus sages. C'est en ce sens que le baiser constitue le cœur de son univers érotique. Ce baiser peut être très fougueux, comme dans l'ode VI $A$ s'amie, où la description du baiser à l'italienne permet à Magny de déployer tout un vocabulaire olfactif et gustatif, utilisant également des motifs empruntés à Jean Second, tel celui des tourterelles se bécotant. Mais la plupart du temps, le comportement de l'amant chez Magny se veut honnête, décent, presque révérencieux, comme dans l'ode III Du ravissement de son ame, où l'amoureux éploré découvre sa dame endormie au bord d'un ruisseau et s'agenouille devant elle pour embrasser ses lèvres.

Chez Magny, comme chez Ronsard, on trouve aussi le simple plaisir de toucher, plaisir qui permet de nommer les membres du corps; dans le geste de l'amant qui palpe, on sent presque celui de l'artiste qui sculpte. Ronsard parle de «tater [la] cuissette, / Ou fesser [la] fesse grossette » (III, 127-28), tandis que Magny se lance dans une longue énumération, où le plaisir tactile n'est pas loin du plaisir lingual :

Et la bouchelette tienne

Couche à plat dessus la mienne,

Laissant folastrer ma main

Soubz le voile de ton sein,

Ore entre tes deux pommettes,

Ore sur tes deux fresettes,

Puis redoublant ces esbatz,

Folastrer encor' plus bas,

Et d'une main plus hardie

Taster ta cuisse arrondie,

Ton ventrelet arrondi,

Et ton petit rebondi... (VI, 93-104)

Cependant, l'art érotique de Magny consiste le plus souvent à faire l'amour de loin, par l'échange, quand l'échange est possible, de baisers chastes et généralement, comme le souligne François Rouget, « de manière indirecte ${ }^{20}$, par l'intermédiaire d'objets ou de fantasmes. Enfin, soulignons la présence chez Magny d'une délicatesse particulière, celle de ne faire « mile petitz jeuz mignards » avec sa «Nymfelette » que durant le jour, «si bien que l'aube vermeille, / Ou Phebus, dès qu'il s'esveille, / Folastrans [les] puisse voir / Du matin jusques au soir » (VI, v. 105-8). Ici encore, la lumière 
du jour vient introduire une distance entre l'amant et sa dame, là où la nuit aurait favorisé la fusion des corps.

\section{Érotisme et poésie}

Malgré les différences relevées jusqu'ici, les érotismes de Ronsard et de Magny partagent cependant certains points communs. Au premier plan est la croyance en la puissance de la poésie dans le cadre des jeux amoureux. Si l'amant ronsardien est constamment frustré dans son désir, son statut de poète lui permet de gagner un certain ascendant sur les jeunes filles. Ainsi, dans la première Folastrie, il avoue qu'il « paye » les faveurs de la maigrelette et de la grasselette « en Chansonnettes, en rymes, et en sornettes » (I, v. 175-76). De la même manière, dans la troisième Folastrie, il fait de sa poésie un instrument de vengeance, destiné à punir Catin la bigote : "Sus donq, pour venger mon esmoy, / Sus, Iämbes, secourez moy, / Venez, Iämbes, sur la teste / De ce luitton, de ceste beste » (III, 157-60). Mais la poésie ne se révèle pas toute-puissante et ne garantit pas à l'amant un triomphe amoureux. Au contraire, le langage du poète peut être contré par le langage de la censure, qui intervient encore pour lui ôter sa maîtresse, soit que c'est « le Tyran de ce village » qui « souille de son langage » (I, v. 180) la grasse et la maigre pucelles, qui « farcist [leurs] noms de blasmes, / D'un mesdire trop amer » (v. 192-93), soit que c'est Catin qui, par ses discours, « ose bien prescher [sa] pucelle, / Pour la convertir ainsi qu'elle / À mille bigotations / Dont elle a mille inventions » (III, v. 99-102).

L'optimisme est plus grand chez Magny : pour lui, la poésie a tous pouvoirs sur l'objet de son désir. Par exemple, dans l'ode XIX, la poésie permet d'obtenir des faveurs de la part de sa dame : qu'elle lui donne un « doulx baiseret » et en retour il fera « un metre, / Qui maugré la rigueur du temps / Éternisera [son] printemps » (v. 74-76); mais si elle se refuse à cet échange, la Muse poétique pourrait bien se courroucer. Magny reprend ici les mêmes thèmes que Ronsard abordait dans les Folastries - la poésie comme monnaie d'échange et comme instrument de punition - mais sur un ton beaucoup plus confiant.

La pièce la plus intéressante à ce titre est l'ode XVIII, dédiée à Ambroise de La Porte, fils de Maurice de La Porte, l'éditeur des Folastries ; cette ode se présente à la fois comme une reconnaissance de l'influence directe de Ronsard, un récit de la genèse du recueil des Gayetez et un éloge de la puissance de la poésie, véritable stimulant et substitut érotique. Dans cette odelette, Magny raconte comment un jour, alors qu'il « folastroit en maincte 
sorte / Avec la Nymfe en qui [il] vit » (v. 8-9), on lui apporta le livret grivois de Ronsard. Aussitôt que le livre entra dans la chambre, " [sa] nymfette refolastra, / Et [lui] soubdain avecques elle / Folastra encor' de plus belle » (v. 18-20). Alors chacun des amants se mit à lire et ils se mirent à « refolastrer » encore plus qu'avant. La volupté éveillée chez les amants est si forte que Magny promet enfin à Ambroise de La Porte de l'inviter la prochaine fois à se joindre à leurs ébats et lui offre en paiement son propre recueil de « gayetez ». Ainsi, le pouvoir de séduction des «doctes folies » de Ronsard est tel qu'il séduit la jeune femme et qu'il provoque un véritable plaisir physique chez l'amant, plaisir qui se transformera à son tour en un autre recueil de vers.

\section{Érotisme dysphorique et anti-érotisme}

À l'intérieur même de ces deux recueils érotiques que sont les Folastries et les Gayetez, il nous semble toutefois voir poindre une méfiance face au désir charnel. Cette méfiance prend chez Ronsard la forme d'un érotisme dysphorique, noir, abreuvé entre autres par la misogynie de l'époque. C'est cette misogynie qui le pousse à souligner l'aspect maléfique et bestial du sexe féminin, "si bien, que quand [il] la perse / [Il] sent les dentz d'une herse, / [Il] entend mill' ossetz cornus, / Qui [lui] blessent les flancs nus » (I, v. 111-14). Il est ici question de la « pucelette maigrelette », mais c'est surtout le personnage de Catin, la courtisane de l'ode III, qui se veut l'incarnation de l'érotisme néfaste. En effet, dès sa jeunesse, alors qu'elle pratique le « mestier [...] où l'un dessus l'autre se veautre », son appétit est si vorace qu'il rend ses amants malades de « la fievre continue » et les mène droit au tombeau ; c'est pourquoi Ronsard la décrit comme « Catin, non jamais soulée / De tuer, pour estre foulée » (III, v. 37-38). Puis, une fois que Catin est convertie, sa paillardise ne diminue pas en force et entraîne toujours des conséquences aussi macabres, puisqu'elle la pousse à hanter les cimetières et à s'y « veautr[er] sus les corps » (III, v. 71) des morts, comme elle le faisait sur celui de ses amants. Ainsi, pour Ronsard, l'érotisme peut aussi comporter sa part de souffrance et de laideur.

Chez Magny, la méfiance envers l'acte charnel prend la forme plus radicale d'un anti-érotisme. Curieusement, au cœur de ce recueil de "gayetez », des accents de chasteté s'élèvent pour condamner l'instinct sexuel. Ce que Magny condamne, en fait, c'est ce même désir qui sous-tend tout le Livret ronsardien, le désir enflammé et dévorant qui ne peut trouver satisfaction. L'auteur lui donne plusieurs dénominations, certaines plutôt neutres, comme 
« ardeur extreme » (III, v. 10) ou « ardeur nompareille» (III, v. 25), d'autres plus négatives, qui soulignent la menace qu'il fait planer sur le principe spirituel de l'homme, comme ce « regret si chauld, qu'il renflame / Tous les sentimens de [son] ame » (VII, v. 11-12), «l'ardeur de [son] ame assaillie » (XXXI, v. 7), ou encore cette « chaleur qui trop [le] maistrise » (XXXVIII, v. 21 $)^{21}$. Mais c'est dans la pièce bachique des Martinales que la condamnation se fait la plus virulente, lorsque Magny encourage son ami Charbonier à laisser s'éteindre son ardeur sensuelle, décrite comme une folie :

\section{Là, là, Charbonier, courage,}

Ceste rage

Qui nous forcene les sens

Pourra bien qu'on n'y travaille,

$\mathrm{Ne}$ te chaille,

$S$ 'alenter avec le tems.

Je veux, Amy, que tu gettes

Jusqu'aux Gettes

Ce soing, acharné mastin... (XXV, v. 265-73)

Pour Magny, comme pour Ronsard, la pulsion érotique peut se révéler dangereuse, lorsqu'elle vient menacer l'équilibre mental de l'individu.

De cette étude sur l'érotisme dans les Folastries et les Gayetez, nous pouvons conclure qu'il n'existe pas un style érotique unique de la Pléiade, qui aurait été initié par Ronsard en 1553 et qui se serait transmis à ses imitateurs. Même chez un disciple aussi immédiat que Magny, l'érotisme change radicalement de physionomie comme de fonction. Il suffit de mentionner une autre œuvre en lien direct avec les Folastries pour s'en convaincre. Dans les Mignardises de Jacques Tahureau, publiées elles aussi en 1554, l'érotisme prend à nouveau une forme différente. Il devient cette fois un monde en lui-même : la liaison sensuelle entre l'amant et sa maîtresse est donnée dès le début et les poèmes grossissent démesurément cette liaison, n'ayant pour fonction que d'en explorer le potentiel érotique, d'en découvrir tous les gestes cachés, de dresser au fur et à mesure les règles des jeux amoureux, dans un cadre bucolique et avec la complicité de la nuit. Et, alors que chez Ronsard et Magny, l'univers érotique était dominé par la figure masculine, la femme ici participe aux ébats au même titre que l'homme et double ainsi les possibilités sensuelles que recèle cette relation.

Ainsi, ce que Ronsard a transmis aux poètes « païens » qui l'ont suivi, c'est un ton poétique - et non un modèle - plus libre, plus franc, lui-même inspiré de la poésie latine et néo-latine et incorporant des éléments des realia 
de l'époque. Cependant, Ronsard est celui qui est allé le plus loin dans l'expression de la gauloiserie. Les Folastries, publiées en 1553, la même année que l'ode $A$ une dame de Du Bellay, signalent un changement dans la sensibilité littéraire de l'époque et dans le style de Ronsard ; le modèle amoureux pétrarquiste commence à se faire trop pesant et l'on sent monter le désir, le temps de quelques recueils, de chanter des passions plus gaillardes, comme celui d'adopter un style plus bas.

\section{Université McGill}

\section{Notes}

1. Pierre de Ronsard, CEuvres complètes, t. 5, Paul Laumonier, éd., Paris, Hachette, 1928, p. XVIII. En outre, Marcel Raymond, dans L'influence de Ronsard sur la poésie française (1550-1585), Genève, Droz, 1965, parlera de Magny comme du « païen de la Brigade » (p. 237).

2. Nous utiliserons l'édition de Laumonier pour les Folastries et, pour Magny, l'édition d'Alistair MacKay des Gayetez, Genève, Droz, 1968.

3. Sans vouloir nous lancer dans de grandes discussions sur la nature de l'érotisme, disons simplement que, pour nous, l'érotisme littéraire se définit comme une représentation littéraire et/ou poétique de l'acte sexuel, c'est-à-dire une représentation linguistique (qui sera abordée surtout dans la première moitié de cet article) et une représentation imaginaire, mettant en image la relation charnelle (abordée dans la deuxième moitié).

4. Voir, par exemple, Jean-Louis Flandrin, Le Sexe et l'Occident. Évolution des attitudes et des comportements, Paris, Seuil, 1981, chapitre 14, p. 279-303.

5. Jean-Marie Goulemot, Ces livres qu'on ne lit que d'une main, Aix-en-Provence, Alinéa, 1991, p. 25.

6. Claude Blum, « Peinture de la souffrance et représentation du moi dans la poésie amoureuse de Ronsard », Sur des vers de Ronsard, 1585-1985, actes du colloque international, Duke University, 11-13 avril 1985, éd. Marcel Tetel, Paris, Aux amateurs de livres, 1990, p. 30-36, remarque le même schéma de désir irréalisable dans les toutes les Amours de Ronsard. Selon lui, « l'objet convoité se dérobe à la saisie » et l'amour se situe donc «à la rencontre d'un puissant désir et d'obstacles qui s'opposent à sa réalisation ». C'est ce qui détermine la représentation de l'amour propre à Ronsard, qui « se dessine sur le fond d'une poétique du manque et de l'insatisfaction » (p. 30).

7. C'est ce désir effréné qui détermine, selon nous, la représentation magnifiée du sexe masculin, souvent tendu dans les Folastries (cf. III, v. 151-54 ; Sonet, v. 1-8).

8. Comment expliquer autrement qu'il ait été publié anonymement? Pour cette question, voir Catharine Randall, "Poetic License, Censorship and the Unrestrained Self : Ronsard's Livret de folastries », Papers on French Seventeenth Century Literature, t. 23 (1996), no 45, p. 449-62.

9. « Voilà comment un beau matin, / Je gaignay dans un beau jardin / Le cueur de ma Nymfe adoree $»($ v. 73-75). 
10. Op. cit., p. X-XI.

11. François Rouget, «Olivier de Magny, amant libertaire et poète de l'inconstance », Revue des Amis de Ronsard, no 11 (juin 1998), p. 106.

12. Sur ce mélange entre une thématique élégiaque et un ton inspiré des vers hendécasyllabiques latins, voir MacKay, op. cit., p. XXVI.

13. Il faut souligner toute la distance qui sépare cette conception de l'érotisme de celle que résume le critique contemporain Roland Barthes, pour qui « l'endroit le plus érotique d'un corps » est « là où le vêtement bâille », là où « la peau (...) scintille entre deux pièces (...), entre deux bords » (Le Plaisir du texte, Paris, Seuil, coll. « Points », 1973, p. 19). Cette conception de l'érotisme est celle du dévoilement, du voyeurisme, de la transgression et elle est selon nous surtout moderne ; on ne trouve pas dans les Folastries cette jouissance qui consiste à dépasser les limites, à aller au-delà des interdits : le plus souvent, le corps s'offre immédiatement nu, tel qui l'est. Pour un contre-exemple, voir le sonnet XXXI des Gayetez, où le poète entrevoit une cuisse blanche sous un vêtement noir et prie la dame de lui dévoiler le reste.

14. À ce propos, Philip Ford note que déjà, dans la poésie érotique latine, les organes génitaux de la femme sont souvent comparés à des fleurs (« Jean Salmon Macrin's Epithalamiorum Liber and the Joys of Conjugal Love », Éros et Priapus. Érotisme et obscénité dans la littérature néo-latine, éd. Ingrid de Smet et Philip Ford, Cahiers d'Humanisme et Renaissance, t. 51, Genève, Droz, 1997, p. 80).

15. Cette description peut s'inspirer de l' anacréontisme, remis à la mode dans ces mêmes années par Henri Estienne, puisqu'il publie dans les premiers mois de 1554 les poésies d'Anacréon (en fait surtout des imitations alexandrines), qu'il avait retrouvées en 1549 lors d'un voyage en Italie. En outre, certaines de ces comparaisons sont déjà, au moment où écrit Magny, passées dans le langage, puisque Giuseppe Di Stefano relève, dans son Dictionnaire des locutions en moyen français, Montréal, CERES (Bibliothèque du Moyen français 1), 1991, la locution «blanc comme lait ».

16. Goulemot, op. cit., p. 55.

17. Il faut remarquer, et cela semble être une caractéristique constante, repérable à travers le temps, de l'érotisme masculin (cf. Francesco Alberoni, L'Érotisme, Paris, Ramsay, 1987, sur la notion de «discontinuité » de l'érotisme masculin), sinon de l'érotisme tout court, que le corps n'est perçu que d'une manière fragmentée. À la manière des blasons anatomiques, on se plaît, proprement, à détailler le corps, à le débiter pour mieux le manipuler. Le poète, dans une posture presque adamique, ne fait que nommer l'un après l'autre les membres à valeur érogène, comme pour les objectiver, les transformer en simples choses, plus utiles que belles.

18. Les références aux Baisers de Jean Second (Les Baisers, éd. Olivier Sers, Paris, Les Belles Lettres, 1996) que l'on retrouve ici sont nombreuses. Il y a, bien sûr, le dénombrement des baisers, procédé lui-même inspiré de Catulle ; les « mille baiseretz » de Magny rappellent l'échange entre Second et Nééra : «Je t'en ai donné mille et en ai reçu mille » (VI, v. 2). Le motif de l'haleine parfumée n'est pas non plus une invention de Second, puisque son origine remonte à l'Anthologie Palatine, mais il utilise pour décrire l'odeur de la bouche des comparaisons que Magny semble avoir reprises presque textuellement : «C'est un nectar, non des baisers, que Nééra / Me verse en son haleine aux suaves parfums, / Elle y 
verse le thym, le nard et la cannelle / Et le miel que l'abeille aux cimes de l'Hymette / Va butiner sur les rosiers en fleur » (IV, v. 1-5). Enfin, l'amante de Charbonier décrit un beau geste passionné (" à ton col branchee, my-panchee... »), mimant en cela l'amante de Second : «Tu te suspends à mon col, Néra, / Tu joins ta bouche adorable à ma lèvre, / Mordant et remordant, râlant sous la morsure » (V, v. 4-6). L'érotisme chez Magny, dans ses moments les plus enflammés, s'inspire peu des élégiaques latins et plutôt des poètes néo-latins, Jean Second au premier plan.

19. Michel Simonin, «Éros aux XVIe et XVIIe siècles : les limites du savoir », Eros in Francia nel Seicento, éd. P.A. Jannini, G. Dotoli et P. Carile, Quaderni del seicento francese, t. 8, Bari, Adriatica ; Paris, Nizet, 1987, p. 29.

20. Rouget, op. cit., p. 106.

21. Dans la vision de Magny, la violence du désir sensuel est telle qu'elle contraint même le Roi des dieux à descendre du ciel pour satisfaire son appétit charnel, pour « nourrir dans son ame / L'ardeur de l'amoureuse flame » (XXXVIII, v. 17-18). 